\title{
A COMPUTER PROGRAMME WITH TEMPERATURE GRADIENT CAPABILITY FOR THE NETWORK ANALYSIS OF HYBRID CIRCUITS
}

\author{
CARL R. ZIMMER \\ Arizona State University, Tempe, Arizona, 85281 USA
}

(Received June 30, 1979)

\begin{abstract}
A modified version of the computer programme SINC-S is described which permits the user to specify independently up to 30 different device temperatures in a given problem when the proper control statement is included. An additional option is an algorithm for the steady-state solution of a non-linear network with periodic inputs, so that realistic system operation may be simulated. The programme may be used to provide more accurate simulation of circuits where large temperature gradients are present, and to furnish input data for other thermal analy sis programmes.
\end{abstract}

\section{INTRODUCTION}

With few exceptions, virtually all modern computeraided design programmes for electronic circuit analysis assume that all network elements, including semiconductor devices, are at the same temperature. However, significant thermal gradients may exist in electronic systems in general and hybrid integrated circuits in particular, so that it would be useful to be able to specify different temperatures for different active devices. This would give more accurate values for the power dissipation in the active devices, which could also be used as input data for computer programmes to give, for example, the temperature distribution in the substrate of a hybrid circuit. Such combined program usage offers the interesting possibility of investigating thermal effects in a proposed design prior to actual circuit fabrication.

\section{PROGRAMME DESCRIPTION}

SINC-S is a modern version of TIME. ${ }^{1}$ It performs d.c. operating point, time domain transient and period steady-state analyses of non-linear electronic circuits containing bipolar transistors, resistors, capacitors, inductors, mutual inductance, and independent current and voltage sources. The bipolar device model used is a modified Ebers-Moll with current-dependent forward beta which may be specified at three collector current values. Included in this model is the temperature dependence of the collector saturation current $I_{s}$, as well as first- and second-order temperature coefficients for forward beta. The programme computes $I_{s}$ at an (absolute) junction temperature $T_{0}$ in accordance with Sze. ${ }^{3}$

$$
I_{s}=A T_{0}^{3} \exp \left(-E_{g 0} / k T_{0}\right)
$$

in which $A$ is a constant dependent upon device material and geometry, $E_{g 0}$ is the band gap energy ( $1.11 \mathrm{eV}$ for silicon) and $k$ is Boltzmann's constant $\left(8.62 \times 10^{-5} \mathrm{eV} / \mathrm{K}\right)$. Similarly, if $\beta_{0}$ is the forward beta at $T_{0}$, it is adjusted to temperature $T$ using the relation

$$
\beta=\beta_{0}\left[1+\operatorname{TC} 1\left(T-T_{0}\right)+\operatorname{TC} 2\left(T-T_{0}\right)^{2}\right]
$$

in which $\mathrm{TC} 1$ and $\mathrm{TC} 2$ are first- and second-order temperature coefficients, respectively. All resistances specified in the programme (including the bulk emitter, base and collector resistances in the device model) may also be characterized by first- and second-order temperature coefficients, and are corrected for temperature in a manner identical to Eq. (2).

\section{PROGRAM OPERATION}

This may be understood by referring to the macro flow chart of Figure 1. The analyses to be performed by the program are controlled by integer flags which may be set by input data statements; those of 


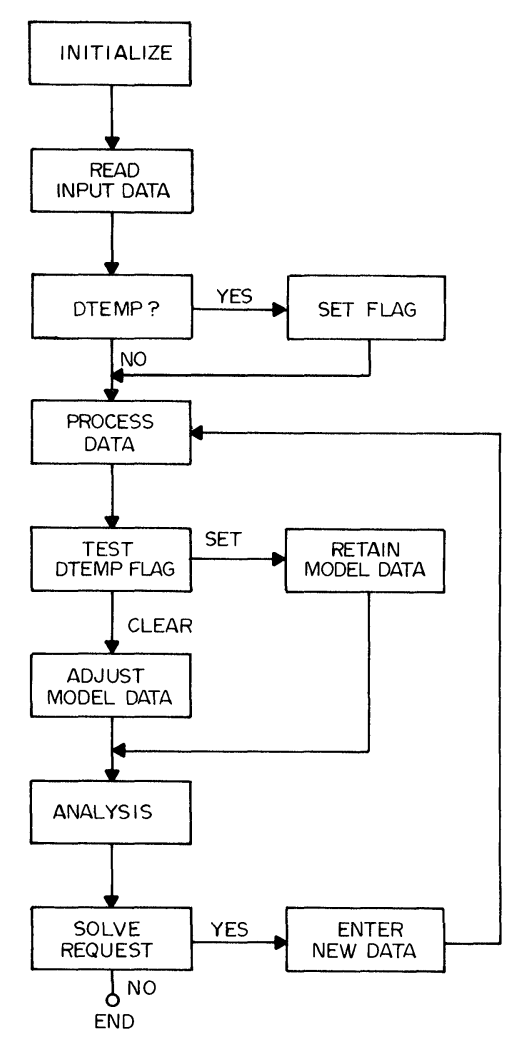

FIGURE 1 Macro flow chart for computer program.

primary interest here are the DTEMP statement, which enables the temperature gradient analysis, and the SOLVE statement, which permits changing up to three data statements without re-entering the rest of the problem and is very helpful in interactive program usage.

\subsection{Normal Program Operation}

In this case a DTEMP control statement is not present in the data. The temperature $T_{0}$ at which device parameters are measured may be given in the device MODEL statement; if absent, a default value of 300 degrees is used. All circuit elements and devices are assumed to be at the same temperature of $T$ degrees Kelvin (this may be specified using a TEMP control statement). If $T$ differs from $T_{0}, I_{s}$ is adjusted using Eq. (1), and forward beta and circuit resistances adjusted using Eq. (2). If a SOLVE request is included, the simulation may be easily repeated for up to three different values of any circuit parameter, but device and "circuit" temperatures will always be the same.

\subsection{Program Operation with DTEMP Statement}

When a DTEMP control statement is included in the data, a corresponding flag is set during the read-in phase of the programme. When that part of the programme using Eqs (1) and (2) is reached, the device parameters are not adjusted, but remain at the values corresponding to $T_{0}$ as specified in the MODEL statement, whereas values for circuit resistances are adjusted to temperature $T$ as before. If a SOLVE request is included, the programmer has the option of obtaining solutions for up to three different values of $T_{0}$, since the DTEMP flag is not cleared until a data end-of-file is reached. This feature is especially helpful when the programme is used interactively from a demand terminal, or when the results are to be used as input data for a thermal analysis program (see below). As SINC-S permits up to 30 sets of model specifications, the user may assign a different junction temperature for up to 30 devices. However, as one set of model parameters may be referenced by more than one device, advantage may be taken of symmetry in the physical layout which indicates that devices would be at the same temperature. The simulations are still restricted in that it is assumed that all circuit resistances are at the same temperature, as mentioned above, but as junction devices are usually the most temperaturesensitive elements it is not felt that this is a serious limitation.

\section{APPLICATIONS}

The programme may be used in conjunction with a substrate thermal analysis programme $\mathrm{e}^{4}$ as follows: an initial set of device temperatures is used as input data, based upon an assumed uniform substrate temperature. The resulting device power dissipations are, in turn, used as input data to the substrate thermal analysis program, and the resultant device temperatures compared with the original assumptions. Using the SOLVE capability, this cycle may then be repeated until reasonable agreement is reached; the number of runs depending upon the thermal sensitivity of the network and the geometry of the hybrid circuit.

Even in those cases where a substrate thermal analysis program is not available, the circuit designer may obtain a more accurate estimate of device and system power input through calculation of junction temperatures using modified thermal impedance concepts ${ }^{4}$ and repeating the simulation with these 
new temperatures as input data. For systems with relatively high power dissipation in one or more devices, such analyses may indicate potential thermal stabilization problems.

It may be argued that the ideal solution would be to have one computer program which simultaneously takes into account both electrical and thermal effects, and indeed such a program ${ }^{5}$ has been implemented. This is a modified version of SPICE which has been used in the design of linear monolithic integrated circuit and voltage regulators. Its major disadvantage is that such simulations typically run ten to twelve times as long as those involving electrical effects d.c. only; as with SINC-S, it is restricted to operating point and time domain transient analyses but will handle different types of semiconductor devices.

\section{CONCLUSION}

A modified version of a computer programme SINC-S has been described which, with suitable control statements, permits the independent specification of up to 30 device temperatures for the more accurate simulation of electronic systems in which significant temperature gradients are present. In the design of hybrid integrated circuits, it may be used alone or in conjunction with a substrate thermal analysis program. At Arizona State University the programme has been implemented on a UNIVAC 1110 system and may be accessed in either batch or demand (timesharing) mode. It has proven to be an effective tool for both instructional and research use, and provides a temperature gradient analysis capability with a minimal increase in program run time and additional required diskfile storage.

\section{REFERENCES}

1. F. S. Jenkins and S. P. Fan, TIME-a nonlinear DC and time-domain simulation program, IEEE J. Solid-State Circuits (August 1971) 182-188.

2. F. N. Colon and T. N. Trick, Fast steady-state periodic analy sis for large-signal electronic circuits, IEEE J. SolidState Circuits (August 1973) 260-269.

3. Sze, S. M., Physics of Semiconductor Devices, John Wiley and Sons, 1969.

4. R. F. David, Computerized Thermal Analysis of Hybrid Circuits, Proc. 1977 Elec. Comp. Conf. (May, 1977) 324-332.

5. P. R. Gray, Computer simulation of integrated circuit performance in the presence of electrothermal interaction, Digest of technical papers of 1976 IEEE SolidState Circuits Conf. (February 1976) 112-113. 

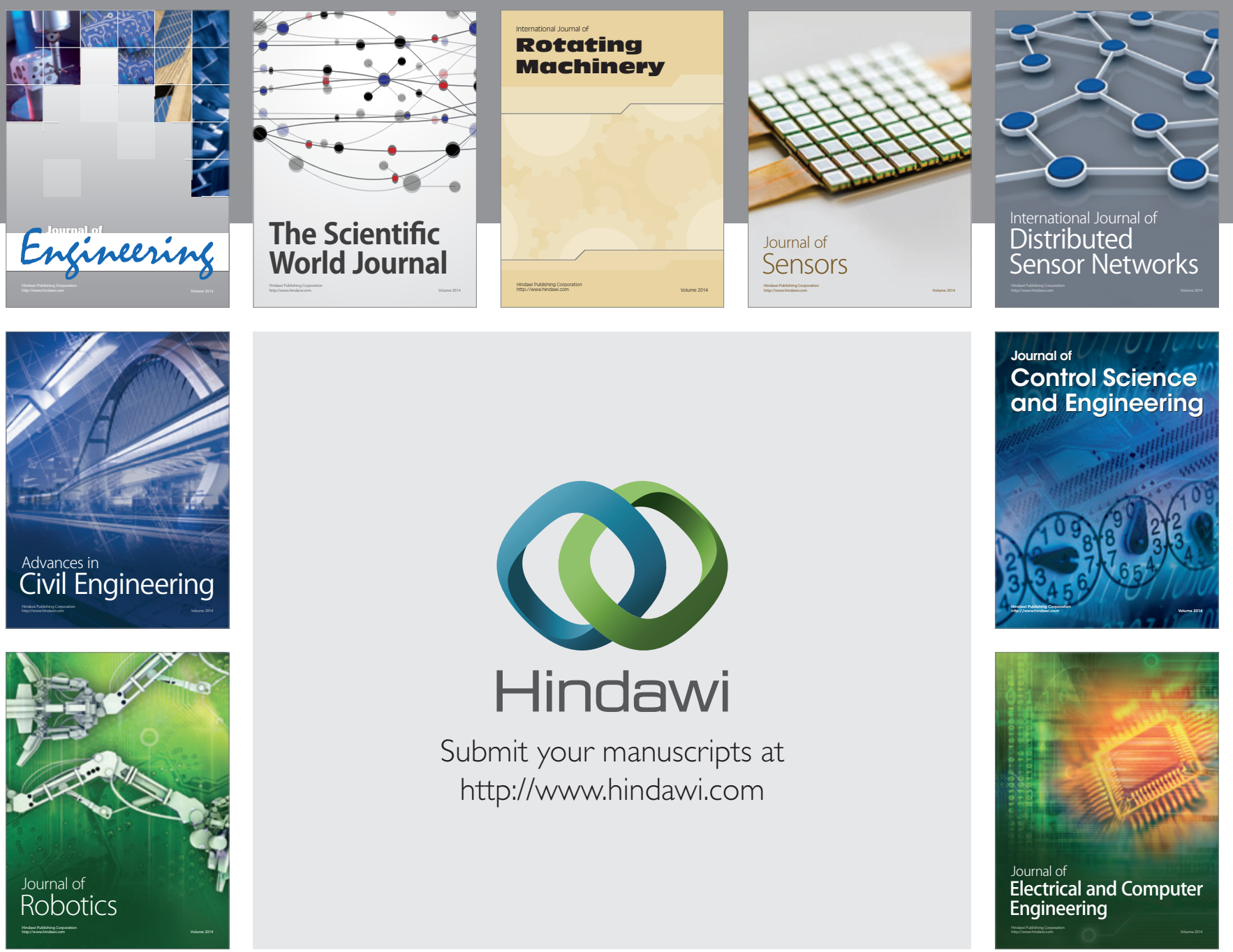

Submit your manuscripts at

http://www.hindawi.com
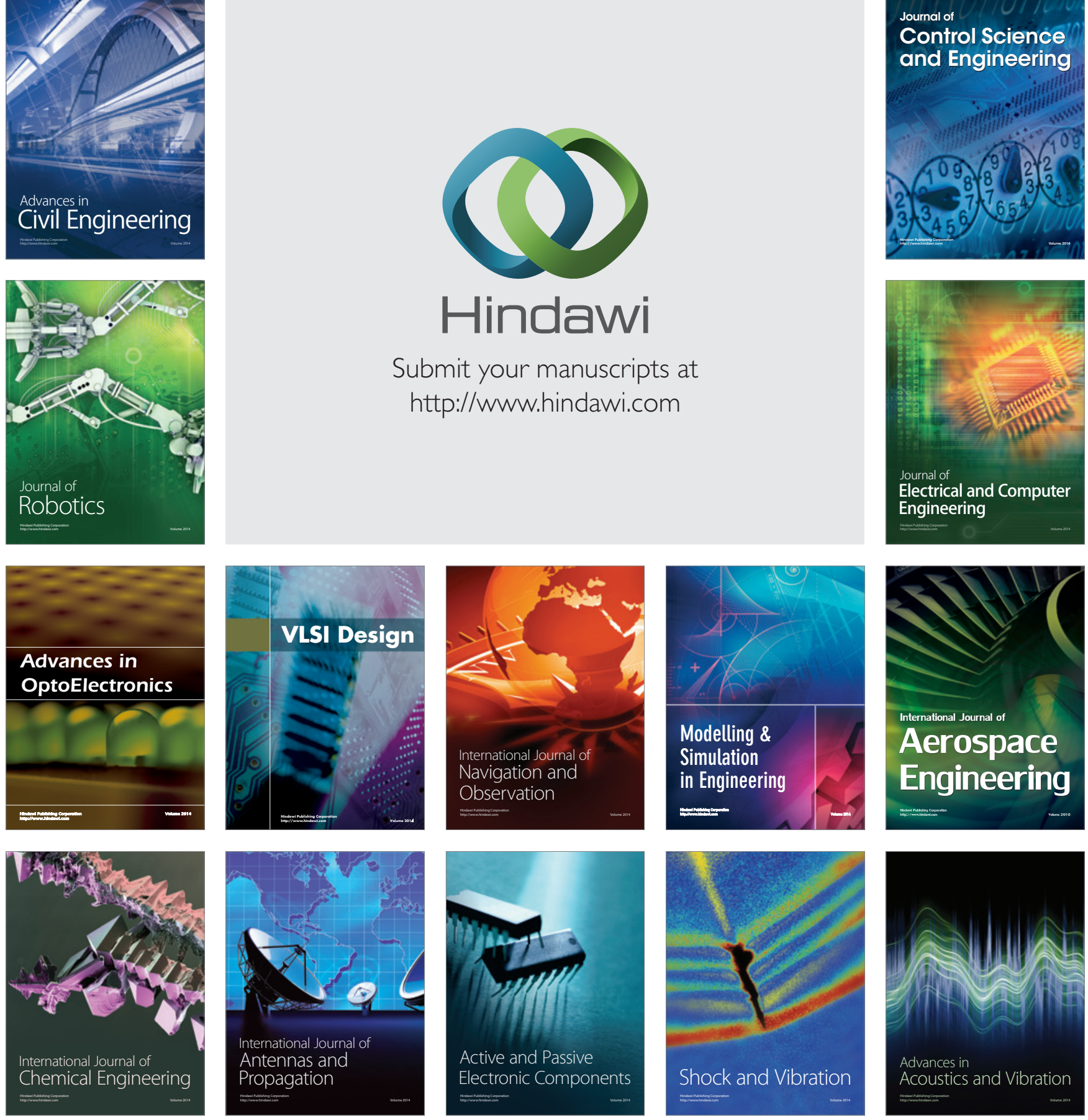